Rev. Elev. Méd. vét. Pays trop., 1978, 31 (1) : 33-37.

\title{
A propos de la cysticercose musculaire des ruminants sauvages et domestiques d'Ethiopie
}

\author{
par M. GRABER $\left(^{*}\right)$
}

\begin{abstract}
RÉSUMẼ
Au cours de deux enquêtes effectuées en Ethiopie durant l'hiver 19761977, dans la province du Sidamo et dans la moyenne vallée de l'Awash (Harrarghe), des cysticerques armés correspondant à Taenia hyaenae de l'hyène et du lycaon ont été isolés des masses musculaires et des organes d'un zébu, d'une gazelle de Soemmering, Gazella soemmeringı (hôte nouveau) et d'un céphalophe couronné, Sylvicapra grimmia.

L'auteur donne des renseignements sur la fréquence relative en Afrique de la ladrerie à cysticerque armé tant chez les ruminants sauvages, où elle est toujours très fréquente, que chez les bovidés domestiques où elle semble sporadique et beaucoup plus irrégulièrement répartie.
\end{abstract}

En Afrique, la cysticercose musculaire des Ruminants sauvages et domestiques se présente sous deux aspects différents.

Dans le premier cas, les vésicules ladres responsables de l'affection sont armées: le scolex est alors pourvu d'un rostre et d'une double couronne de crochets en poignard, alternants et inégaux. Ces cysticerques correspondent à quatre espèces au moins de Taeniidae parasites de l'intestin des carnivores sauvages (6, 17) : Taenia acinonyxi Ortlepp, 1938 du guépard (Acinonyx jubatus) et de la Panthère (Panthera pardus) ; Taenia crocutae Mettrick et BeverleyBurton, 1961 des hyènes (Crocuta crocuta, l'hyène tachetée ; Hyaena hyaena, l'hyène rayée ; Hyaena brunnea, l'hyène brune) ; Taenia gonyamai Ortlepp, $1938 \mathrm{du}$ lion (Panthera leo) et du guépard; Taenia hyaenae Baer, 1924 des hyènes et du lycaon (Lycaon pictus).

Dans le second cas, le cysticerque est inerme, sans rostre, ni crochets. L'espèce en cause est Cysticercus bovis dont le Taenia adulte, Taenia saginata Goeze, 1782 vit dans l'intestin grêle de l'homme.

(*) Chaire de Parasitologie, Ecole Nationale Vétérinaire, Marcy l'Etoile, 69260 Charbonnères-les-Bains.
Les deux types de cysticerques sont capables d'affecter indifféremment tous les ruminants. Malheureusement, encore aujourd'hui, on ne possède que peu de renseignements (7) sur la fréquence relative, chez le zébu, des cysticerques armés et, chez les ruminants sauvages, de Cysticercus bovis.

La réunion F. A. O./U. N. E. P./W. H. O. de Nairobi $\left({ }^{*}\right)$ a insisté particulièrement pour qu'en la matière, des enquêtes précises soient effectuées en différents points d'Afrique.

Dans cette optique, il a paru intéressant de donner le résultat de quelques observations faites en Ethiopie au cours de l'hiver 1976-1977.

\section{MATÉRIEL ET MÉTHODE}

Chez le zébu, les récoltes ont été effectuées à l'abattoir de Wondo-Genet (Shashamane, Sidamo) sur des animaux originaires du Borana (Sud-Sidamo), stockés et engraissés pendant près de quatre mois à la « Quarantaine », Ranch annexe de l'abattoir.

(*) Joint F. A. O./U. N. E. P./W. H. O. consultation on field control of taeniasis and echinococcosis, Nairobi, Kenya, juin 1976. 
Vingt-cinq animaux adultes ont été examinés et 83 cysticerques présentant l'aspect habituel de vésicules, remplis d'un liquide incolore on tété recueillis, ouverts et éclaircis au lactophénol. Les scolex ont été sectionnés à la lame de rasoir et les coupes ainsi obtenues ont été placées entre lame et lamelle, légèrement écrasées et examinées au microscope.

Parallèlement, toujours sur la concession de la "Quarantaine », 4 hyènes ont été autopsiées. Les cestodes présents dans l'intestin ont été isolés. Les strobiles ont été colorés au carmin chlorhydrique, tandis que les scolex subissaient le même traitement que les cysticerques prélevés chez les zébus.

Par ailleurs, dans le cadre d'une enquête sur la pathologie de la faune sauvage (14), 11 ruminants ont été tués dans la moyenne Awash (Harrarghe), à savoir : 4 Nyala de montagne, Tragelaphus buxtoni ; 1 Oréotrague, Oreotragus oreotragus; 1 Bushbuck, Tragelaphus sp.; 1 Duiker, Sylvicapra grimmia; 2 Oryx, Oryx beisa et 2 gazelles de Soemmering, Gazella soemmeringi.

L'examen attentif des carcasses a permis de découvrir quelques cysticerques musculaires qui, bien qu'ayant subi partiellement la dégénérescence caséo-calcaire, ont pu être identifiés de la même façon que précédemment.

\section{RÉSULTATS}

1) Chez les zébus, 82 des 83 parasites examinés se sont révélés être des cysticerques inermes appartenant à l'espèce Cysticercus bovis. Un seul d'entre eux était pourvu d'une double couronne de 36 crochets en forme de poignard et placés en alternance. Les plus grands qui mesurent 216 à $228 \mu$ (moyenne, $223,7 \mu$ ) sont munis d'un manche large, droit et plus long que la lame. La garde est entière. Le bord postérieur présente, dans son tiers inférieur, une encoche suivie d'un léger renflement. Les plus petits (150-156 $\mu$, moyenne, 151,5 $\mu$ ) ont le manche plus court que la lame qui est fortement recourbée. La garde est bifide.

La question s'est immédiatement posée de savoir à quel Taenia des carnivores sauvages pouvait se rapporter ce parasite. Les caractères des crochets et leurs dimensions permettent d'éliminer d'emblée Taenia acinonyxi, Taenia gonyamai et Taenia crocutae (17). Le seul Cestode qui s'en rapproche le plus est sans conteste Taenia hyaenae dont le cycle évolutif a été bien étudié en Somalie par PELLEGRINI $(11,13)$ : l'hôte définitif est l'hyène et l'hôte intermédiaire un bovidé domestique ou sauvage $(6,9,10,12)$.

Sur les 4 hyènes sacrifiées à la « Quarantaine », 2 d'entre elles hébergeaient Taenia hyaenae (un seul exemplaire par animal). L'examen des scolex a permis de dénombrer 36 à 38 crochets mesurant 210-216 $\mu$ (moyenne, 212,5 $\mu$ ) pour les plus longs et 138 à $150 \mu$ (moyenne, $143 \mu$ ) pour les plus courts. Dans les segments gravides, le nombre de branches utérines varie de 8 à 12 .

Sur d'autres exemplaires de Taenia hyaenae provenant d'une hyène tuée à Debré-Zeit (Shoa) en 1975, la longueur des crochets (34) est légèrement supérieure : 215 à $221 \mu$ et 141 à $153 \mu$.

2. Chez les ruminants sauvages de la moyenne Awash, des cysticerques armés ont également été rencontrés à 2 reprises différentes: dans le foie d'une gazelle de Soemmering, dans le cœur et les muscles d'un duiker.

Le nombre ( 36 à 38 ), les caractères et les dimensions des crochets (grands : 204 à $228 \mu$, moyenne, $220 \mu$; petits : 135 à $162 \mu$, moyenne, $146,5 \mu$ ) laissent penser que, là encore, le Taenia adulte correspondant pourrait être Taenia hyaenae.

\section{COMMENTAIRES}

1) En Ethiopie, Cysticercus bovis n'est pas le seul responsable des cas de ladrerie rencontrés. A côté, il existe - tant chez les ruminants domestiques que chez les ruminants sauvages des cysticerques armés à localisation musculaire dont la forme adulte se trouve dans l'intestin grêle des hyènes et des lycaons.

Les résultats des deux enquêtes menées dans la vallée de l'Awash et la province du Sidamo confirment donc entièrement d'autres observations faites, il y a déjà quelques années, en Erythrée $(1,2,4,5)$ et dans le Shoa (3) où ce type de cysticerque affecte le zébu et certaines antilopes, notamment le Céphalophe couronné, Sylvicapra grimmia.

2) La cysticercose musculaire des ruminants sauvages semble, jusqu'à plus ample informé, devoir être imputée à des cestodes de carnivores. 
Ce sont, en Ethiopie, essentiellement des Hyaenidae, très nombreux dans ce pays et souvent porteurs de Taenia (1 fois sur 2).

Il s'agit là d'un phénomène général que l'on retrouve dans un grand nombre de réserves et de zones cynégétiques de l'Est $(15,16)$, du Sud et du Centre de l'Afrique $(6,7)$ : ainsi, en Tanzanie (parc de Serengeti), dans les muscles de 205 ruminants sauvages reconnus ladres, aucun cysticerque inerme n'a pu être mis en évidence, mais uniquement des cysticerques armés ayant pour origine des cestodes de lion (Taenia gonyamai) ou de hyène (Taenia crocutae).

$\mathrm{Au}$ Tchad, sur 22 cas de ladrerie décelés chez diverses antilopes, 18 d'entre eux sont le fait de cysticerques armés de type Taenia hyaenae. Quant à la ladrerie à Cysticercus bovis, elle est exceptionnelle chez les Antilopes d'Afrique centrale et ce genre d'infestation ne se produit qu'à la faveur de circonstances qui nécessitent un contact étroit entre des individus porteurs de Taenia saginata et des animaux non chassés, vivant à proximité de l'homme (7).

3) La ladrerie des zébus éthiopiens est, la plupart du temps, à base de Cysticercus bovis. Toutefois, la présence dans les muscles et dans certains organes de cysticerques armés n'est pas très rare. Il est, dans l'état actuel de nos connaissances, impossible de donner des pourcentages d'infestation précis. A Wondo-Genet, sur 25 animaux parasités, un seul d'entre eux présentait ce type de cestode, ce qui est bien inférieur aux chiffres figurant dans la statistique de $\mathrm{CO}$ CEANI (5) valable pour certaines parties de l'Erythrée :

Nombre de zébus autopsiés : 531 .

Nombre de zébus parasités : 155 , soit 29,19 p. 100.

- Par Cysticercus bovis : 99 ; soit 63,8 p. 100.

- Par cysticerque armé : 32, soit 20,6 p. 100.

- Infestations mixtes : 24 , soit 15,6 p. 100 .

Les enquêtes mériteraient d'être reprises sur une plus vaste échelle au niveau de tous les grands abattoirs de la République éthiopienne. C'est ce qu'a d'ailleurs prévu le projet «Parasitic zoonoses: Taeniasis, Cysticercosis-Echinococcosis, Hydatidosis " qui a été discuté lors de la réunion «Animal disease investigation and research coordination committee - $»$ tenue à Addis-Abéda le 21.3.1977, réunion qui s'inscrit dans un cadre plus général de lutte contre ces zoonoses dans le monde (voir supra).
Dans les pays voisins, notamment en Somalie, on retrouve cette même disposition, les pourcentages variant sensiblement selon les abattoirs. PELLEGRINI, à Mogadiscio, observe que 24 p. 100 des dromadaires et 10 p. 100 des zébus sont porteurs de cysticerques armés, tandis qu'à Kismayu (1), le taux d'infestation n'est plus que de 1,5 p. 100 .

En ce qui concerne les autres pays d'Afrıque, on ne possède actuellement que peu de renseignements valables. La présence de cysticerques armés a été signalée chez des bovins au Masailand, Kenya (16), ainsi qu'au Tchad, chez des dromadaires et des zébus, mais uniquement dans l'Est du pays, le long de la frontière du Soudan (Ouaddaî). Le taux d'infestation est faible : 1,1 p. 100 des 176 animaux autopsiés au cours de l'année 1958.

Il est curieux de constater que la ladrerie à cysticerque armé des bovidés domestiques se voit surtout dans des zones de plateau ou de montagne, à végétation herbacée peu abondante et où les populations de hyènes sont très importantes. Celles-ci s'infestent en dévorant les cadavres d'animaux parasités morts de maladies ou de misère physiologique engendrée par une sous-alimentation chronique. Les bovidés, souvent carencés et, de ce fait, atteints de pica, se contaminent en absorbant les œufs et les anneaux de Taenia répandus sur le sol par leurs hôtes. $\mathrm{Ce}$ contact étroit entre hyènes et bétail domestique s'établit au voisinage des points d'eau, plus rarement autour des villages ou des lieux de pâture.

Lorsque ces conditions ne sont pas remplies - notamment dans les zones plus humides à couverture herbacée plus dense où les bovins vivent dans de meilleures conditions - la présence de cystîcerques armés est beaucoup plus rare, voire même fortuite. C'est le cas des animaux qui ont fait l'objet de cette observation. Originaires du Borana où la cysticercose bovine et le téniasis humain paraissent moins répandus que partout ailleurs en Ethiopie (*), ils ont parcouru à pied la distance comprise entre Yavello et Awassa, c'est-à-dire toute la zone caféière de Dilla, avant d'être entreposés sur la concession de la «Quarantaine » pendant environ 14 semai-

(*) Les habitants du Borana, contrairement aux autres Ethiopiens, ne consomment la viande que très cuite, ce qui restreint les chances d'infestation (Dr FESSEHA, Communication personnelle). 
nes. Celle-ci est entièrement clôturée et le peuplement humain est réduit aux bergers chargés de la surveillance des troupeaux. Par contre, les hyènes y pullulent, surtout de part et d'autre de la zone boisée et marécageuse qui traverse de part en part la concession et elles hébergent fréquemment Taenia hyaenae.

On ne sait où les bovins autopsiés ont contracté la cysticercose, peut-être sur la concession, plus vraisemblablement au cours de leur déplacement. Toutefois, compte tenu de la présence possible chez le même animal des 2 espèces de cysticerques et du temps passé à la «Quarantaine ", temps amplement suffisant pour que les vésicules ladres acquises sur place puissent être facilement mises en évidence, on aurait dû à l'examen rencontrer un nombre beaucoup plus élevé de cysticerques armés, ce qui n'est pas le cas.

L'infestation des bovins domestiques par des cysticerques provenant de Taenias de carnivores sauvages semble obéir à certaines règles qui, là encore - et en dehors des zones cygénétiques demanderaient à être mieux précisées.

4) La ladrerie à cysticerque armé n'est pas dangereuse pour la santé de l'homme, puisque ce dernier n'intervient pas directement dans le cycle évolutif.

En ce qui concerne les ruminants sauvages, les carcasses atteintes de cysticercose ont un caractère répugnant et ne peuvent être livrées à la consommation. Si les taux d'infestation sont élevés, les pertes peuvent être importantes et condamner, dans certains cas, les projets de mise en valeur rationnelle du potentiel économique que représente la faune sauvage.

Chez le zébu, il n'est pas possible à l'œil nu de distinguer la ladrerie à cysticerque armé de la ladrerie à cysticerque inerme : l'inspecteur les confond toutes deux. Pour en limiter l'incidence, dans les régions d'élevage où la cysticercose à Taenia armé représente un pourcentage non négligeable des saisies totales, il serait souhaitable de s'attaquer à l'hôte définitif, l'hyène, et d'en réduire le nombre, ce qui diminuerait d'autant les possibilités de contamination du bétail domestique.

\section{CONCLUSIONS}

Les observations faites en Ethiopie au cours de l'hiver 1976-1977 dans la moyenne Awash (Harrarghe) et à l'abattoir de Wondo-Genet (Sidamo) confirment la présence dans les muscles et les organes du zébu et de divers ruminants sauvages de cysticerques armés pouvant être rapportés à Taenia hyaenae Baer, 1924, cestode que l'on rencontre fréquemment dans l'intestin des hyènes sacrifiées.

De ces 2 observations très limitées, il serait hasardeux de tirer des conclusions définitives : les enquêtes devront être poursuivies à une plus grande échelle et dans un plus grand nombre de provinces.

\section{Remerciements}

Nous tenons à remercier vivement $M$. le Docteur GOUALO qui nous a aimablement ouvert les portes de l'abattoir de Mondo-Genet, ainsi que Messieurs les assistants qui ont procédé aux récoltes de parasites.

Nos remerciements iront également à M. BLANC et PETITCLERC qui nous ont confié la détermination des Cestodes et des Trématodes récoltés chez les ruminants sauvages de la vallée de l'Awash.

\section{SUMMARY}

\section{Muscular cysticercosis of ethiopian wild and domestic ruminants}

During the winter 1976-1977, investigation into cysticercosis of game animals killed in Awash valley (Ethiopia, Harrarghe) indicates that the Cysticerci recovered from the musculature and organs of a Duiker, Sylvicapra grimmia and of a Soemmering's Gazelle, Gazella Soemmeringi are the larval stages of a Tapeworm, Taenia hyaenae Baer, 1924 parasitizing spotted Hyaena (Crocuta crocuta) and wild dog (Lycaon pictus).

Soemmering 's Gazelle is a new host.

In Sidamo, the same parasite affects also zebu cattle, but the rate of infestation seems very low.

The author gives some informations on incidence in Africa of armed cysticerci which are most frequently found in Antelopes, whereas, in domestic cattle, they seem sporadic and irregularly distributed. 


\section{RESUMEN}

A propósito de la cisticercosis muscular de los rumiantes salvajes y domésticos de Etiopia

Durante dos encuestas efectuadas en Etiopia durante el invierno 1976-1977 en la provincia de Sidamo y en el valle medio de Awash (Harrarghe), se aislaron cisticercos armados correspondiendo a Taenia hyaenae de la hiena y del licaón a partır de masas musculares y órganos de un cebú, de una gacela de Soemmering, Gazella soemmeringi (huesped nuevo) y de un cefalofo coronado, Sylvicapra grimmia.

El autor da informes sobre la frecuencia relativa en Africa de la cisticercosis con cisticerco armado en los rumiantes salvajes donde siempre es muy frecuente y en los bóvidos domésticos donde parece esporádica y mucho más irregularmente repartida.

\section{BIBLIOGRAPHIE}

1. ANGELOTTI (S.). Contributo casistico sul Cysticercus dromedarii Pellegrini, 1945. Boll. Soc. ital. Med. Ig. trop., Eritrea, 1947, 7 (5/6) : 544-549.

2. BATTELLI (C.). Il Cysticercus dromedarii Pellegrini, 1945 in Eritrea. Boll. Soc. ital. Med. Ig. trop., 1949, 9 (3/4) : 289-294.

3. BERGEON (P.). A veterinary parasitology survey. Report to the Government of Ethiopia, Rome, F. A. O., 1968,38 p. (No T. A. 2458).

4. CALL (C.). Il Cysticercus dromedariii Pellegrini, 1945 in um Antilope Eritrea. Boll. Soc, ital. Med. Ig. trop., 1949, 9 (3/4): 300-302.

5. COCEANI (C.). Frequenza del Cysticercus bovis e del Cysticercus dromedarii tagli zebu eritrei. Boll. Soc. ital. Med. Ig. trop., Eritrea, 1949, 9 (3/4) : 295299.

6. GRABER (M.), TRONCY (P. M.), THAL (J.). La cysticercose musculaire des Ruminants sauvages d'Afrique Centrale. Rev. Elev. Méd. vét. Pays trop., 1973, 26 (2) : 203-220.

7. GRABER (M.). La ladrerie a Cysticercus bovis des Ruminants sauvages de la République du Tchad. Bull. epizoot. Dis. Afr., 1974, 22 (4) : 357-360.

8. GRABER (M.). Helminthes et helminthiases des animaux domestiques et sauvages d'Ethiopie. Maisons-Alfort, I. E. M. V. T., 1976, t. II, 206 p.

9. PELLEGRINI (D.). Il «Cysticercus dromedarii n. sp. » nel cammello e relativa Cysticercosi. Boll.
Soc. Ital. Med. Ig. trop., Eritrea, 1947, 7 (3/4) : $317-$ 324.

10. PELLEGRINI (D.). Il «Cysticercus dromedarii n. sp. » nel bovino. Boll. Soc. Ital. Med. Ig. trop., 1947, 7 (5/6) : 550-553.

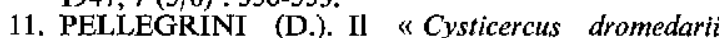
Pellegrini, $1945 》$ e lo stato larvale della Taenia hyaenae Baer, 1924. Boll. Soc. ital. Med. Ig. trop., Eritrea, 1947, 7 (5/6) : 554-565.

12. PELLEGRINI (D.). Il "Cysticercus dromedarii n. sp. » nella capra. Boll. Soc. ital. Med. Ig. trop., Eritrea, 1949, 8 (3/4) : 172-175.

13. PELLEGRINI (D.). Le Cysticercus dromedarii du chameau et des bovins (Pellegrini, 1945) et le Taenia hyaenae correspondant de l'hyène (Baer, 1924). Bull. Off. int. Epizoot., 1961, 32 (4) : 257-261.

14. PETITCLERC (M.). Awash (Ethiopie) : collecte d'informations sur la pathologie des animaux sauvages. Rapport. Maisons-Alfort, I. E. M. V. T., 1977, 53 p.

15. SACHS (R.). Untersuchungen zur artbestimmung und differenzierung des Muskelfinnen ostrafrikanischer Wiltiere. Tropenmed. Parasit., 1969, 20 (1): 39-50.

16. SACHS (R.). Cysticercosis of East african game animals. Jl. S. Afr. vet. med. Ass., 1970, 41 (2) : 79-85.

17. VERSTER (A.). A taxonomic Jevision of the genus Taenia Linneaus, $1758 \mathrm{~s}$. str. Onderstepoort J. vet. Res., 1969, $36(1): 3-58$. 